\title{
THE PROCESS OF BECOMING SUSPICIOUS OF ULTERIOR MOTIVES
}

\author{
Miquelle A. G. Marchand and Roos Vonk \\ Radboud University Nijmegen
}

We examined the process of becoming suspicious and discovering ulterior motives. Participants read about a likable behavior, then sequentially received ten cues about potential ulterior motives of the actor. Participants were asked to think aloud while they were reading. Their thoughts were coded. We expected that the general impression of the actor would gradually become more negative, whereas suspicion would first increase and later decrease, concomitant with increased certainty that ulterior motives were indeed involved. Confirming our hypotheses, we found a linear effect for general impression and a quadratic effect for suspicion. Discussion focuses on the development of suspicion as a process and on the relevance of our findings to other settings in which multiple hypotheses are entertained.

There is ample evidence that most people cannot distinguish honest from deceptive behavior of others (Anderson, DePaulo, Ansfield, Tickle, \& Green, 1999; DePaulo \& Friedman, 1998; Ekman \& O'Sullivan, 1991; Malone \& DePaulo, 2001). One of the reasons why people are so poor at detecting deception is a pervasive truthfulness bias: People tend to accept everything they see at face value. Gilbert, Tafarodi, and Malone (1993) proposed that people cannot comprehend something without accepting it as true (cf. Gilbert, 1991). People do have the power to assent, reject, and to suspend their judgment, but only after they have initially believed the information to which they have been exposed.

\footnotetext{
This research was supported by NWO grant 575-12.030/1. We would like to thank Steven Fein and Laurie Rudman for their helpful comments and suggestions.

Address correspondence to Miquelle Marchand, CentERdata, P.O. Box 90153, 5000 LE Tilburg, The Netherlands. E-mail: M.Marchand@uvt.nl
} 
Gilbert et al. (1993) provided evidence that " ... belief is first, easy, and inexorable and that doubt is retroactive, difficult, and only occasionally successful" (p. 231). As a consequence, most people tend to judge others as truthful most of the time (O'Sullivan, Ekman, \& Friesen, 1988; Zuckerman, Fischer, Osmun, \& Winkler, 1987; Zuckerman, Koestner, Colella, \& Alton, 1984).

Related research on attribution suggests that a disposition is often directly inferred from behavior, whereas the role of other variables (such as the situation) is underestimated or ignored by perceivers (known as the correspondence bias or fundamental attribution error; Gilbert \& Malone, 1995; Ross \& Nisbett, 1991). This very robust phenomenon, again, reflects the tendency to take behavior at face value. More recent research by O'Sullivan (2003) suggests that the fundamental attribution error significantly undermines the ability to detect honesty and deception accurately. She found evidence that when observers thought positively about someone, they also tended to believe the other person was telling the truth even when the person was lying. Overcoming this robust effect appears to be difficult both in attribution research and deception detection: "The tendency to judge other people on the basis of enduring traits, rather than situational relevant states, is one of the reasons most lie catchers are so inaccurate, and adjusting this cognitive heuristic is not easy" (O'Sullivan, 2003; p. 1325).

There is one particular circumstance in which the truthfulness bias can be overcome, namely, when a perceiver becomes suspicious about a person's motives (Fein, 1996). For example, imagine that you are reading about John who takes a colleague with car trouble to the garage. Probably you think of John as a nice, helpful man. However, when you learn that the colleague is an attractive woman, that John is not very helpful in other situations, and that he has no serious relationship at the moment, you might start wondering whether John took her to the garage merely because he was interested in her romantically. In this case, ambiguity emerges about the actor's motives; the perceiver does not know whether the behavior should be ascribed to a correspondent trait (helpful) or to an ulterior motive (romantically interested).

Fein (1996) describes suspicion as a state in which perceivers hold multiple, rival hypotheses about the motives or sincerity of the actor's behavior. According to Hilton, Fein, and Miller (1993), 
perceivers in this state suspend their judgment about the actor until they have more information about the actor's motives. If they have to give a judgment, their evaluation will be neutral. This was also found in a study conducted by Vonk (1999a), in which likable behaviors enacted toward superiors were judged more moderately than toward subordinates, indicating that subjects took into account the possibility of ulterior motives (i.e., "brownnosing"). Further, it seems that suspicious perceivers think actively and systematically about why the actor behaved as he or she did. This is what Fein (1996) calls the attributional mindset. Concomitant with this thoughtfulness and moderate judgments, the correspondence bias is reduced, because looking beyond face value requires an analytical state of mind.

In Fein and his colleagues' studies, suspicion of ulterior motives is experimentally induced. Participants receive a description of a situation that puts them in a state of suspicion. Subsequently, additional information is provided that can change participants' judgments in one direction or the other. For example, Fein, Hilton, and Miller (1990) had participants read a story about a man who courts a wealthy widow. Participants were unwilling to conclude either that he was in love with her or that he was after her money; they were not certain which inference to make (i.e., they were suspicious). Then they read additional information about the man's visit to a grocery store, where the clerk gave him too much change. In one condition, the man returned the extra money, while in the other condition he kept the extra change. Participants who read that the suitor returned the money concluded that he truly loved the woman, while the other participants concluded that he was motivated by greed. It is important to note that in our view, at this point participants cannot be regarded as suspicious anymore: They no longer question whether the actor is insincere; they know. ${ }^{1}$

Although it is evident that suspicion about ulterior motives can overcome the correspondence bias, it is not clear how the process

1. According to the definition, suspicion has ended when a perceiver does not hold multiple hypotheses anymore, and is confident that the actor was driven by ulterior motives. At this point, the actor is no longer a "suspect", but is "guilty". This does not mean that having been suspicious may not produce subsequent residual effects. We will return to this issue in the Discussion. 
of suspicion evolves. In the studies by Fein and colleagues, participants are either in a state of suspicion (upon reading a story about an actor who behaves suspiciously) or out of that state (upon receiving subsequent information that corroborates one of the two possible inferences). Presumably, however, perceivers often do not instantly become suspicious, nor do they instantly abandon suspicion when they receive disambiguating information (unless it is extremely diagnostic). In the present study, we sought to extend the research by Fein et al. by examining the entire process, from the moment that perceivers have no suspicion at all, through a phase in which suspicion emerges, to the moment that they become certain that the actor indeed has an ulterior motive. We hypothesized that perceivers become suspicious gradually-as they start questioning a person's motives-and that they abandon suspicion gradually, as more information becomes available that confirms their suspicion.

In order to record this dynamic process, we first presented participants with a description of a positive behavior, so they would have a positive evaluation of the actor (e.g., taking a colleague with car trouble to the garage). Then participants received small pieces of additional information (cues) that imply that the actor might have an ulterior motive (e.g., the colleague is a woman; the colleague is attractive). We asked participants to think out loud after each cue (cf. Greenwald, 1968; Petty \& Cacioppo, 1981), and we coded their thoughts on two dimensions: evaluation and suspicion.

Our hypotheses are that participants' general impressions of the actor will become increasingly negative as more cues are encountered, whereas suspicion will first increase and then decrease, as participants become more certain of their judgment and change their initial evaluations (from positive to negative). In short, we expect a linear trend for evaluation and a quadratic trend for suspicion.

\section{STUDY 1}

METHOD

Participants. Forty-nine students at Radboud University Nijmegen participated for a fee of $€ 1.00$ (at the time, about \$1.10). 
Two participants were dismissed, one due to stuttering and one because he did not think aloud the whole time.

Stimulus Materials. In order to safeguard the generalizability of the results, we used three different scenarios (presented between participants), which were pretested among 59 participants. From the data of this pretest, we also selected ten cues per scenario, which were all judged to be moderately informative on a seven-point scale. More extreme informative cues were not selected, because one highly informative cue could be sufficient to create certainty about ulterior motives. The first scenario was: John asks Henrietta to marry him. Examples of cues for this scenario include: "John likes luxurious products"; "Henrietta is not very interesting"; "Henrietta is a widow." The mean for the ten cues in this scenario was $M=3.56$ ( $1=$ not at all informative, 7 = very informative).

The second scenario was: John takes a colleague with car trouble to the garage. Examples of cues for this scenario include: "the colleague is a woman"; "John does not have a steady relationship"; "John offers his colleague compliments about her work." The mean informativeness of the ten cues was $M=3.99$.

In the third scenario, the main sentence was: "John responds enthusiastically to another person's idea during the meeting." Examples of cues include: "the other person is higher in rank"; "John does not have tenure"; "John will soon have an assessment." The mean informativeness for the ten cues used in this scenario was $M=3.91$.

In addition to the specific actor cues, negative general actor cues of John were used, for example, "John does not offer his seat to the pregnant woman on the bus." This type of behavior descriptions, in other settings, was also used in studies by Fein et al. (1990).

For each scenario, ten cues were presented. For order variation, these were divided into three blocks each containing three or four sentences. Each block contained one cue about the target (e.g., the other person is higher in rank), one was a negative general actor cue, and one was a specific actor cue (e.g., "John does not have a tenure"). Both the order in which the blocks were presented, as well as the order of the sentences within each block, were randomized. This way, we ensured that no more than two "similar" sentences (e.g., two general actor cues) were presented sequentially. Also, in the car trouble scenario, the first cue was always that the colleague was a woman, because some other cues, (e.g., 
the colleague is attractive) were ambiguous without this information. In the meeting scenario, the first two cues were always that the person with whom John agrees is a man and that the man is higher in rank. This was done to prevent redundancy with a later cue, that this man is John's superior. ${ }^{2}$

Procedure. Participants sat behind a computer in a cubicle. The experimenter sat near them. She told participants that they would participate in a study in which they had to think aloud, and that their thoughts would be recorded with a tape recorder. The participants then read an instruction on the computer screen, in which it was explained that they had to form an impression of John and that they had to express their thoughts aloud. They were asked to click "ok" in order to receive the scenario sentence (for example, "John asks Henrietta to marry him"), which had to be read aloud immediately, after which they started thinking aloud. In the same way, participants clicked "ok" for every next cue, read it aloud, and expressed their thoughts.

Thoughts after each cue were rated for evaluation of the actor (ranging from $-2=$ negative to $+2=$ positive) and for how suspicious the participant was. A suspicion score of 3 was given for maximum suspicion (i.e., at least two possible motives for the behavior were considered and the participant could not decide between these two). A score of 2 was given when the participant did consider two explanations for the behavior, but leaned toward one of the two and thought the other less likely. A score of 1 was given when the participant was certain either that the actor was nice/friendly or that he had an ulterior motive. Two judges coded the thinking aloud protocols independently of each other for half of the participants. The thoughts were coded in their original order, because in some cases participants said that a piece of extra information did not change their views; in that case the thought was rated identical to the previous thought. The correlations between the scores of both judges were $r=.81$ for evaluation and $r=$ .79 for suspicion.

The judges also coded at which particular cue a participant started doubting the actor's motives $(r=.87)$ and at which cue the

2. The exact randomization scheme is available upon request. 
participant concluded for certain that the actor had an ulterior motive $(r=.84)$.

\section{RESULTS AND DISCUSSION}

A MANOVA with cue (0-10) as within-subjects factor and scenario as between-subjects factor showed that, with regard to evaluation, the expected linear trend emerged, $F(1,44)=147.31, p<.01$. Regardless of scenario (interaction $F(2,44)=1.35, n s$ ), the actor was judged less positively when more cues were presented. The means are shown in Figure 1. There was also a significant quadratic trend for this variable, $F(1,44)=91.21, p<.01$. This is due to the fact that the evaluation first declines rapidly, but later levels off, which results in a deviation from the perfect linear pattern.

With regard to suspicion, we found the expected quadratic trend, $F(1,44)=14.14, p<.01$. The linear trend was not significant $F(1,44)$ $=1.95, n s$. The means in Figure 2 indicate that initially, after reading the scenario sentence, the participants were certain of their judgments of the actor, then quickly started doubting those judgments; gradually they tended to become less suspicious, until at the end they were certain about the motives again. As the figure also indicates, there is an interaction effect with scenario $F(2,44)=12.80, p<$ .01. It appears that there was no suspicion in the marriage proposal scenario. More detailed examination of the thinking aloud protocols in this condition suggests that the process often developed so quickly that it could not be coded. For example, at the cue that Henrietta is a widow, one participant immediately said: "Oh, widow, I think she is a rich woman. John himself has a low income evidently." The maximum suspicion score of 3 was never reached here, because the participant immediately changed from a positive to a negative image of the actor.

With regard to the question at which cue suspicion arises, no clear pattern was found for the marriage proposal scenario. Many participants (9 out of 12), however, became suspicious at the cues "Henrietta is not very interesting" (four participants) or "Henrietta is not very attractive" (five participants). This means that suspicion emerged at varying order positions, because the order of cues was varied. When participants first read that 


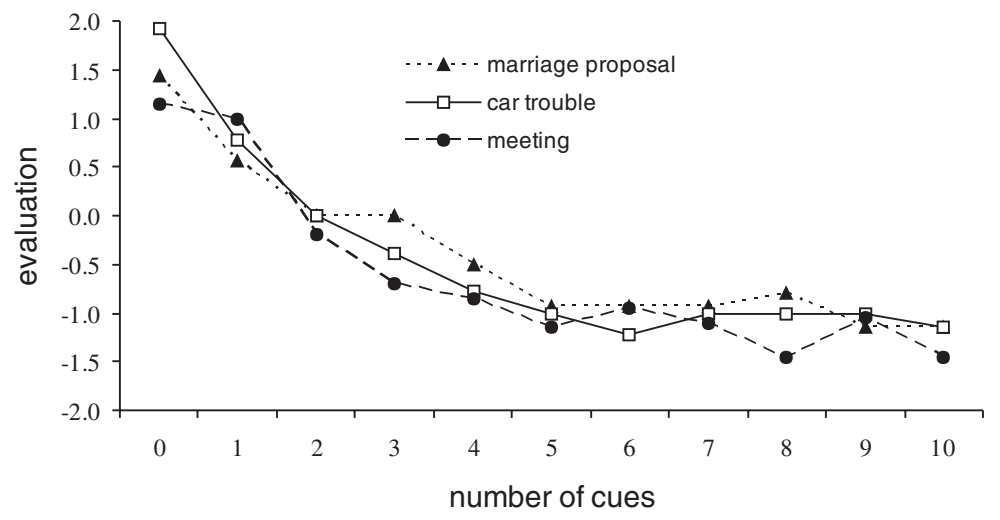

FIGURE 1. Evaluation for each scenario at cue 0-10 $(-2=$ negative $+2=$ positive $)$ in Study 1.

Henrietta is not very attractive they expect that John wants to marry her because of her inner qualities. When they learn subsequently that she is not very interesting, they are certain that there has to be an ulterior motive. Conversely, when she is dull, participants assume she is attractive.

In the car trouble scenario most participants (12 out of 13) became suspicious at the first cue (the colleague is a woman). In the meeting scenario, 17 out of 21 participants became suspicious at the second cue (the person with whom John agrees is higher in rank). This implies that the rapid increase in suspicion in these scenarios might be caused by the fact that they always started with fixed cues that happened to evoke suspicion in most of the participants (see Figure 2). In order to examine this possibility we conducted the second study.

\section{STUDY 2}

\section{METHOD}

In this study we replicated the meeting scenario (John responds enthusiastically to another person's idea during the meeting), but 


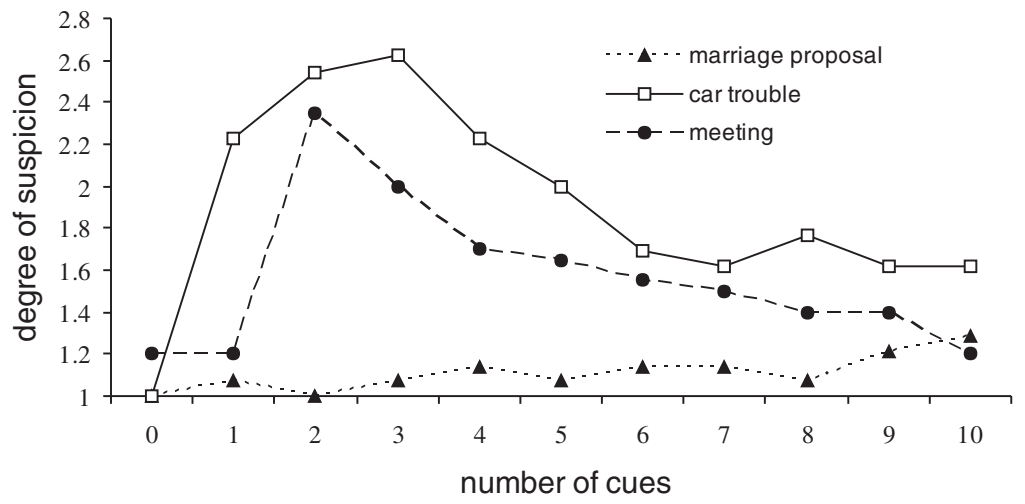

FIGURE 2. Degree of suspicion for each scenario at cue $0-10(1=$ certainty, $3=$ suspicion $)$ in Study 1.

presented all cues in random order. The cue "the person with whom John agrees is his superior" was removed and the cue "the other person is higher in rank" was not presented at a fixed location. In other respects, the method used was the same as in Study 1. Thirteen participants from Radboud University Nijmegen participated for a fee of $€ 1.00$.

\section{RESULTS AND DISCUSSION}

The data were coded by the same independent judges. ${ }^{3}$ The results again indicated a linear trend for evaluation, $F(1,12)=41.15$,

3. In addition, we asked two student coders, who were uninformed about our hypotheses, to rate both evaluation and level of suspicion for the thinking aloud data from Studies 1 and 2 . The coders showed acceptable agreement (all $r s>.60, p s<.01$ ). For the first three scenarios from Study 1, the analyses of the data produced by these judges showed the same patterns: a linear trend for evaluation $F(1,32)=79.64, p<.01$ and a quadratic trend for suspicion $F(1,32)=5.51, p=.03$. For the random meeting scenario from Study 2 , the results also replicate the patterns found earlier: a linear trend for evaluation $F(1,12)=21.63, p<.01$ and a quadratic trend for suspicion $F(1,12)=6.48, p<.03$. 
$p<.01$. Also, a quadratic trend for suspicion emerged, $F(1,12)=$ $5.97, p<.03$, whereas the linear trend was not significant, $F(1,12)=$ $1.65, n s$. As can be seen in Figure 3 , the increase of suspicion in this study is weaker and more gradual. Thus, the quick increase in the first study was probably caused by the fact that a relatively informative cue, that the other person is higher in $\operatorname{rank}(M=4.75$, in pretest, as compared to $M=3.82$ for other cues), was the second cue for every participant. Presumably, the same applies to the car trouble scenario and the cue that the other person is a woman.

\section{GENERAL DISCUSSION}

Extant research suggests that people are not good at detecting insincerity, due to the correspondence bias and a more general truthfulness bias (O'Sullivan, 2003). A suspicious mindset is a quite effective antidote to these biases (Fein, 1996). The present research uniquely showed how this mindset emerges and progresses as more information is received. In addition, each study showed that people can be quite ready to suspect ulterior motives. Participants read one of three different scenario descriptions of an actor who behaved in a positive way, to induce an initially favorable correspondent inference. Then they received additional, moderately informative cues that evoked suspicion by casting a negative light on the behavior. As predicted, thinking-aloud protocols showed the expected linear effect for evaluation of the actor. That is, participants evaluated the actor less positively as more information was presented. In addition, they also showed the expected quadratic effect for suspicion. Perceivers who were initially certain of their evaluation soon began to doubt the actor's motives (suspicion). As more information was presented, they became progressively more certain that the actor indeed had ulterior motives, until finally they were certain. Taken together, these findings demonstrate that suspicion is a dynamic process that unfolds over time as people grapple with the possibility that an actor has ulterior motives, and then become convinced. As more information is processed, the negative opinion of the actor increases, while uncertainty rises and then falls.

Further, our findings suggest that participants can detect ulte- 


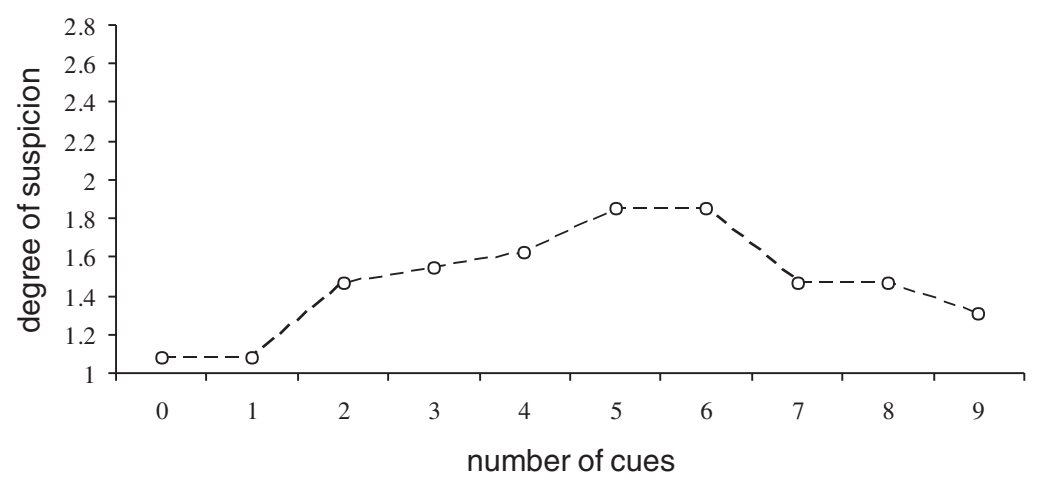

FIGURE 3. Degree of suspicion for the random meeting scenario at cue $0-9(1=$ certainty, $3=$ suspicion) in Study 2.

rior motives or insincerity quite quickly, even though they were presented with cues that were moderate in informativeness. The results also indicate that certain cues effectively trigger suspicion. Mainly, these cues involve characteristics of the person toward whom the behavior is enacted (i.e., target cues or actor-target relationship cues), such as "the other person is a woman," "the other person is higher in rank," and "the other person is not attractive." In line with Vonk's (1999a) studies, in which participants evaluated an actor's positive behavior differentially depending on the target's hierarchical position, the largest changes in suspicion and evaluation emerged on the basis of target information. By comparison, the general actor cues used by Fein et al. (1990) had a minor effect on suspicion in our studies. In only four out of 120 instances, general actor cues led to suspicion or to certainty that the actor had an ulterior motive. The difference between our actor cues and those used by Fein et al. is that ours were only informative on a general evaluative dimension and were unrelated to the behavior (e.g., in the wealthy widow scenario, offering a seat to a pregnant woman only means that the actor is friendly; it is not related to honesty or greed). It is possible 
that the actor cues in Fein's studies were more disambiguating because they were descriptively related to honesty or to the trait dimension under consideration (e.g., in the wealthy widow scenario, returning extra change to a clerk is related to honesty as well as greed, so this cue helped participants conclude that the actor was in love with the widow).

In general, cues that are specifically related to the characteristic under consideration probably evoke greater changes in suspicion. In our studies, these were often target cues (e.g., in the wealthy widow scenario, the woman is not very interesting). Note that the influential role of target information is at odds with the correspondence bias and the tendency to focus on the actor and ignore the behavioral field. It is, however, in accord with recent evidence (Ham \& Vonk, in preparation) that characteristics of the targets of behavior are automatically included in spontaneous trait inferences (e.g., helping a colleague activates different traits than helping a superior).

In our studies, the informativeness of the cues presented was held constant. In real life, cues are probably more differentiated in their level of informativeness. Moreover, the cues would not be observed in such a short amount of time. In everyday life, people learn a few things when they meet a person and they learn something new on a different occasion. Although the basic mechanisms should be the same, the process of becoming suspicious and then certain may take more time outside of the lab. Also, in real life, people not only receive negative information about others, but also positive information, which might further slow down the process of suspicion or even reverse it.

In the present studies, the information was presented to participants. In real life, when people become suspicious about an actor's motives, they may themselves start to search for relevant information that could disambiguate the behavior, although we have evidence that the information they acquire is not the most diagnostic (Marchand \& Vonk, 2004). Nonetheless, future research should examine a variety of protocols designed to mirror information gathering under more natural conditions.

In addition, individual differences may affect the speed of the suspicion process. For example, people who are high in need for closure (i.e., have a desire to reduce ambiguity [Kruglanski, 1989] 
and tend to be impulsive and form quick judgments of others [Kruglanski \& Webster, 1996]) may tend to think of others as primarily either good or bad. If so, participants with a higher need for closure may be quicker to jump through the process and conclude that an actor is insincere. Of course, they might also be slower to start doubting people if they prefer to retain their original, positive evaluation.

If people are certain about their judgment of an actor, they can no longer be described as suspicious, because they are not entertaining multiple, rival hypotheses anymore. At that point, by definition, suspicion is over. Interestingly, Fein (1996) has shown that when perceivers have been suspicious about an actor's motives, they are less likely to fall prey to the correspondence bias when making subsequent judgments about a novel actor. While this might suggest that suspicion continues onward, we think this result can be explained by a priming or accessibility effect. That is, once a suspicious mindset has been activated, it is more likely to be triggered again in new situations.

It is important to note that the process of suspicion may apply to any situation in which a person's behavior can be guided by multiple motives, thus causing perceivers to hold multiple hypotheses, or doubt whether their initial judgment was correct. This may occur, for instance, in persuasion settings (e.g., a salesman claiming that this is the best buy ever, or any persuasive message from a biased source; cf. Campbell, 1995), deception detection situations (e.g., a woman who finds lipstick on her husband's collar), self-presentational situations (e.g., a person claiming high competence in a job interview; Leary, 1995; Vonk, 1999b), and even the detection of discrimination, in which case mixed motives are also involved (e.g., a person may be rejected for a job either because of sexism or because she is not adequate; Fiske, Bersoff, Bordiga, Deaux, \& Heilman, 1991; Berkvens \& Vonk, 2002). Our studies are a first step toward mapping the challenging and fascinating process of becoming suspicious, in person perception as well as other domains. 


\section{REFERENCES}

Anderson, D. E., DePaulo, B. M., Ansfield, M. E., Tickle, J. J., \& Green, E. (1999). Beliefs about cues to deception: Mindless stereotypes or untapped wisdom. Journal of Nonverbal Behavior, 23, 66-89.

Berkvens, M., \& Vonk, R. (2002). Horen, zien en zwijgen: De effecten van covariatie- informatie op de waarneming van seksisme door vrouwen [The effects of covariance information on the perception of sexism by women]. In E. van Dijk, E. Kluwer, \& D. Wigboldus (Eds.), Jaarboek Sociale Psychologie 2002 (pp. 25-32). Delft, The Netherlands: Eburon.

Campbell, M. C. (1995). When attention-getting advertising tactics elicit consumer inferences of manipulative intent: The importance of balancing benefits and investments. Journal of Consumer Psychology, 4, 225-254.

DePaulo, B. M., \& Friedman, H. S. (1998). Nonverbal communication. In D. T. Gilbert, S. T. Fiske, \& G. Lindsey (Eds.), The handbook of social psychology (Vol. 2, pp. 3-40). Boston: McGraw-Hill.

Ekman, P., \& O'Sullivan, M. (1991). Who can catch a liar? American Psychologist, $46,913-920$.

Fein, S. (1996). Effects of suspicion on attributional thinking and the correspondence bias. Journal of Personality and Social Psychology, 70, 1164-1184.

Fein, S., Hilton, J. L., \& Miller, D. T. (1990). Suspicion of ulterior motivation and the correspondence bias. Journal of Personality and Social Psychology, 58, 753-764.

Fiske, S. T., Bersoff, D. N., Borgida, E., Deaux, K., \& Heilman, M. E. (1991). Social science research on trial: Use of sex stereotyping research in Price Waterhouse v. Hopkins. American Psychologist, 46, 1049-1060.

Gilbert, D. T. (1991). How mental systems believe. American Psychologist, 46, 107-119.

Gilbert, D. T., \& Malone, P. S. (1995). The correspondence bias. Psychological Bulletin, 117, 21-38.

Gilbert, D. T., Tafarodi, R. W., \& Malone, P. S. (1993). You can't not believe everything you read. Journal of Personality and Social Psychology, 65, 221-233.

Greenwald, A. G. (1968). Cognitive learning, cognitive response to persuasion, and attitude change. In A. G. Greenwald, T. C. Brock, \& T. M. Ostrom (Eds.), Psychological foundations of attitudes (pp. 147-170). San Diego, CA: Academic Press.

Ham, J., \& Vonk, R. (in preparation). Spontaneous slime inferences.

Hilton, J. L., Fein, S., \& Miller, D. T. (1993). Suspicion and dispositional inference. Personality and Social Psychology Bulletin, 19, 501-512.

Kruglanski, A. W. (1989). Lay epistemics and human knowledge: Cognitive and motivational bases. New York: Plenum Press.

Kruglanski, A. W., \& Webster, D. M. (1996). Motivated closing of the mind: "Seizing" and "freezing." Psychological Review, 103, 263-283.

Leary, M. R. (1995). Self-presentation: Impression management and interpersonal behavior. Madison, WI: Brown \& Benchmark.

Malone, B. E., \& DePaulo, B. M. (2001). Measuring sensitivity to deception. In J. 
A. Hall \& F. J. Bernieri (Eds.), Interpersonal Sensitivity: Theory and measurement (pp. 103-124). Mahwah, NJ: Lawrence Erlbaum.

Marchand, M. A. G., \& Vonk, R. (under review). Motivated by vanity, not insanity: Target-observer differences in information search and impression revision after ingratiation.

O'Sullivan, M. (2003). The fundamental attribution error in detecting deception: The boy-who-cried-wolf effect. Personality and Social Psychology Bulletin, 29, 1316-1327.

O'Sullivan, M., Ekman, P., \& Friesen, W. V. (1988). The effect of comparisons on detecting deceit. Journal of Nonverbal Behavior, 12, 203-215.

Petty, R. E., \& Cacioppo, J. T. (1981). Attitudes and persuasion: Classic and contemporary approaches: Dubuque, IA: Wm. C. Brown Company Publishers.

Ross, L., \& Nisbett, R. E. (1991). The person and the situation: Perspectives of social psychology. New York: McGraw-Hill.

Vonk, R. (1999a). Differential evaluations of likable and dislikable behaviours enacted towards superiors and subordinates. European Journal of Social Psychology, 29, 139-146.

Vonk, R. (1999b). Impression formation and impression management: Motives, traits, and likeability inferred from self-promoting and self-deprecating behavior. Social Cognition, 17, 390-412.

Zuckerman, M., Fischer, S. A., Osmun, R. W., \& Winkler, B. A. (1987). Anchoring in lie detection revisited. Journal of Nonverbal Behavior, 11, 4-12.

Zuckerman, M., Koestner, R., Colella, M. J., \& Alton, A. O. (1984). Anchoring in the detection of deception and leakage. Journal of Personality and Social Psychology, 47, 301-311. 